\title{
Sonographic Evaluation of Umbilical Vein
}

\author{
Deval Shah ${ }^{1} \cdot$ Nilpa Shah $^{1}$
}

Received: 21 February 2018/Accepted: 21 April 2018/Published online: 10 May 2018

(C) The Author(s) 2018

\begin{abstract}
To evaluate intraabdominal course of umbilical vein, its relation with portal vein and ductus venosus and its anomalies. All pregnant women were examined during midtrimester anomaly scan between January 2015 and December 2017. With evaluation of umbilical vein in its intraabdominal course, size of umbilical vein, its relation to the stomach and the gallbladder and origin of ductus venosus were studied in middle and upper abdominal axial and sagittal planes by two dimensional and colour doppler sonography. Anastomosis of umbilical vein with portal vein and its further course was examined and detailed anatomical survey was made to look for associated anomalies. Fetal and neonatal outcome was assessed after delivery. Twelve cases of umbilical vein abnormalities were evaluated. Of them, there were three cases of umbilical vein varix, four cases of persistent right umbilical vein and five cases of abnormal umbilical-portal-systemic venous shunt were noted. Examination of the intraabdominal course of umbilical vein and ductus venosus should be a part of second trimester anomaly scan. Proper knowledge of umbilical portal venous system is mandatory.
\end{abstract}

Keywords Ductus venosus (DV) · Persistent right umbilical vein (PRUV) · Umbilical vein (UV) · Umbilicalportal-systemic venous shunt (UPSVS) - Umbilical vein varix (UVV)

Deval Shah

devalnilpa@gmail.com

1 Astha Hospital and Fetal Medicine Center, Deesa, Gujarat, India

\section{Introduction}

The umbilical vein (UV) carries oxygenated blood from the placenta to the fetus. UV, Portal vein (PV) and ductus venosus (DV) are an integrate part of fetal circulation. For better understanding, knowledge of embryology of the venous system is necessary. According to Yagel et al. [1], two horns of sinus venosus receive three pairs of veins at embryonic four weeks: 1 . The cardinal veins from the body of the embryo, 2. the UVs from the placenta and 3. the vitelline veins (VV) from the yolk sac. These veins open into the corresponding horns of sinus venosus. Simultaneously, liver starts developing in the area of septum transversum. The parts of these veins nearest to the heart are embedded into septum transversum, and undergo considerable changes. Anastomotic network develops between both VV in the area of liver that forms liver capillaries. Both UVs get connected to this capillary network. Some part of these UVs and VVs anastomose and some regress. Right UV atrophies, some hepatic sinusoids enlarge and form direct passage to connect left UV to proximal IVC. This forms the DV. Anastomosis between the two VVs forms the portal venous system.

\section{Anatomy of Umbilical Vein}

The left UV enters into the fetal abdomen at cord insertion. As mentioned by Yagel et al. [1], during intrahepatic course, it connects to left portal vein (LPV) (Fig. 1x, y). LPV gives three branches to the left lobe of the liver. LPV continues as portal sinus (PS), and gives rise to DV. PS turns to right where it joins main portal vein (MPV). MPV divides into right anterior and posterior PVs and supplies the right lobe of liver. So, during fetal life, LPV is wider and flow is in the opposite direction. DV drains into IVC 
Fig. 1 Schematic drawings (x, y) of umbilical-portal-ductus venosus vascular unit, twodimensional images $(\mathbf{a}, \mathbf{b}$, c) correspond to planes $\mathbf{a}-\mathbf{c}$; colour doppler image

(d) correspond to plane (d). Ao, aorta; DV, ductus venosus; GB, gall bladder; $\mathrm{H}$, heart; $\mathrm{HV}$, hepatic veins; IVC, inferior vena cava; LPV, left portal vein; MPV, main portal vein; RAPV, right anterior portal vein; RPPV, right posterior portal vein; $S$ stomach; Sp spine; UV, umbilical vein
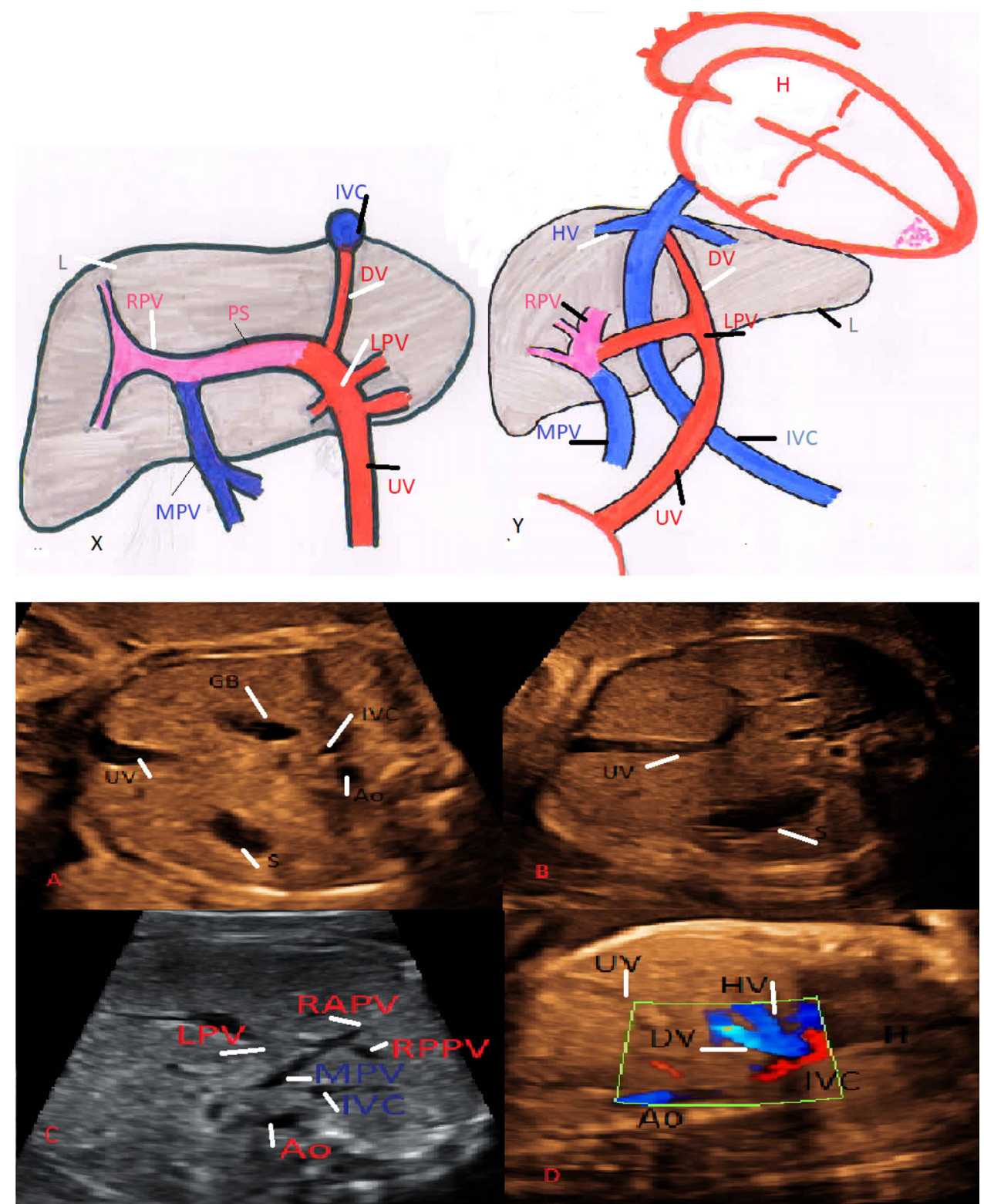

just distal to its connection to the right atrium. IVC, three hepatic veins and DV drain into the subdiaphragmatic vestibulum at the base of the right atrium.

The aim of our study was to diagnose anomalies related to $\mathrm{UV}$, we diagnosed three cases of umbilical vein varix (UVV), four cases of persistent right umbilical vein (PRUV) and five cases of abnormal umbilical portal systemic venous shunt (UPSVS).

\section{Methods}

Pregnant women during midtrimester anomaly scan between January 2015 and December 2017 were examined. The courses of UV, PV and DV in axial and sagittal views of abdomen by two dimension and colour doppler sonography were studied. UV was examined in the middle and upper abdominal axial planes. Middle abdominal axial view (plane A) (Fig. 1a) at cord insertion and upper abdominal axial view (Plane B) (Fig. 1b) for anastomosis of UV with left portal vein (LPV). In the same planes, relationship of UV with stomach and gall bladder was assessed. Anatomy of umbilical portal venous system that also includes portal sinus, main portal vein and right portal vein was studied by sliding and rotating from upper abdominal axial plane (plane C) (Fig. 1c). Origin of DV was examined in sagittal plane (plane D) (Fig. 1d). Plane $\mathrm{A}, \mathrm{B}$ and $\mathrm{D}$ were examined in all fetuses while plane $\mathrm{C}$ only when abnormality was noted. In cases where DV was not seen, connection of UV to PV was documented and 
alternative drainage site of UV were searched and also intrahepatic portal venous system was studied. Detailed examination of these fetuses was done to rule out other anomalies. Two cases detected in third trimester were also included. Growth of these fetuses was monitored and final outcome was documented. Cases with anomalies like anterior abdominal wall defects, congenital diaphragmatic hernia, heterotaxy syndrome where anatomy of upper abdominal venous system is disturbed were not included.

\section{Results}

We were able to identify normal umbilical portal venous system in nearly all cases. In very few cases where gall bladder was not found, we identified left or right UV on the basis of its curve. During this period, total 12 anomalies (Table 1) of UV were diagnosed: three cases of UVV, four cases of PRUV and five cases of UPSVS anomaly.

\section{Umbilical Vein Varix}

UVV is focal dilatation of UV (Fig. 2). We found three cases of intraabdominal UVV (case 1-3). 1st case was primigravida examined first time at 36 weeks of gestation, cystic swelling of $13 \mathrm{~mm}$ with high velocity flow was seen in middle abdomen, other findings were normal for that fetus. She delivered a healthy male child without complication; this child is now 18-month-old and healthy. 2nd case was seen at 30 weeks with bilateral multicystic dysplastic kidneys. Intraabdominal extrahepatic $12 \mathrm{~mm}$ UVV was associated finding. 3rd case was seen at 20 weeks, with $8 \mathrm{~mm}$ intraabdominal extrahepatic UVV, no associated anomalies were seen. That delivered IUGR fetus.

\section{Persistent Right Umbilical Vein}

During embryonic period, right UV regresses and left UV remains to carry oxygenated blood from placenta. Left UV joins LPV and turns to right, making curve opposite to stomach (Fig. 3). Failure of RUV involution results into PRUV anomalies. We found four cases of PRUV (case 4-7). Two cases were seen with normal situs, 1st case was associated with single umbilical artery, the outcome was normal. 3rd and 4th cases were seen with situs inversus totalis with normal heart. We detected right UV in 3rd case that is normal association with situs inversus totalis and in 4th case, we found left UV.

\section{Abnormal Umbilical Portal Systemic Venous Shunt}

UV joins left portal vein (LPV) during intrahepatic course, this LPV continue as portal sinus (PS). PS gives rise to ductus venosus (DV) (Fig. 1x, y). We found five cases

Table 1 List of 12 cases of anomalies of umbilical vein

\begin{tabular}{llllll}
\hline Number & Diagnosis & GA (weeks) & Association & Intrahepatic portal system & Outcome \\
\hline 1 & UVV & 36 & - & Normal & L \& H \\
2 & UVV & 30 & Bilateral MCDK & Normal & Delivered PT \\
3 & UVV & 20 & IUGR on F/U & Normal & L \& H \\
4 & PRUV & 18 & SUA & Normal & L \& H \\
5 & PRUV & 20 & LBW on F/U & Normal & L \& H \\
6 & PRUV & 22 & Situs inversus totalis ${ }^{\text {a }}$ & Normal & L \& H \\
7 & PRUV & 22 & Situs inversus totalis & Normal & Not yet delivered \\
8 & AUPSVS ADV with UV to IVC & 18 & Cardiomegaly, hydrops & Abnormal & TOP \\
9 & AUPSVS ADV with UV to IVC & 24 & Cardiomegaly, hydrops & Abnormal & IUFD \\
10 & AUPSVS ADV with UV to RA & 18 & Cardiomegaly, hydrops & Abnormal & TOP \\
11 & AUPSVS ADV with RPPV to IVC & 18 & LBW on F/U & Normal & L \& H \\
12 & AUPSVS ADV & - & Normal & L \& H
\end{tabular}

$A D V$ agenesis of ductus venosus, AUPSVS abnormal umbilical portal systemic venosus shunt, $F / U$ follow up, IUGR intrauterine growth retardation, IUFD intrauterine fetal death, IVC inferior vena cava, $L \& H$ live and healthy, $L B W$ low birth weight, $M C D K$ multicystic dysplastic kidney, $P R U V$ persistent right umbilical vein, $P T$ preterm, $R A$ right atrium, $R P P V$ right posterior portal vein, $S U A$ single umbilical artery, $T O P$ termination of pregnancy, $U V V$ umbilical vein varix

${ }^{\mathrm{a}}$ Situs inversus totalis with PRUV

${ }^{\mathrm{b}}$ Situs inversus totalis with LUV

${ }^{\mathrm{c}}$ Connection of intrahepatic portal systemic shunt not found 


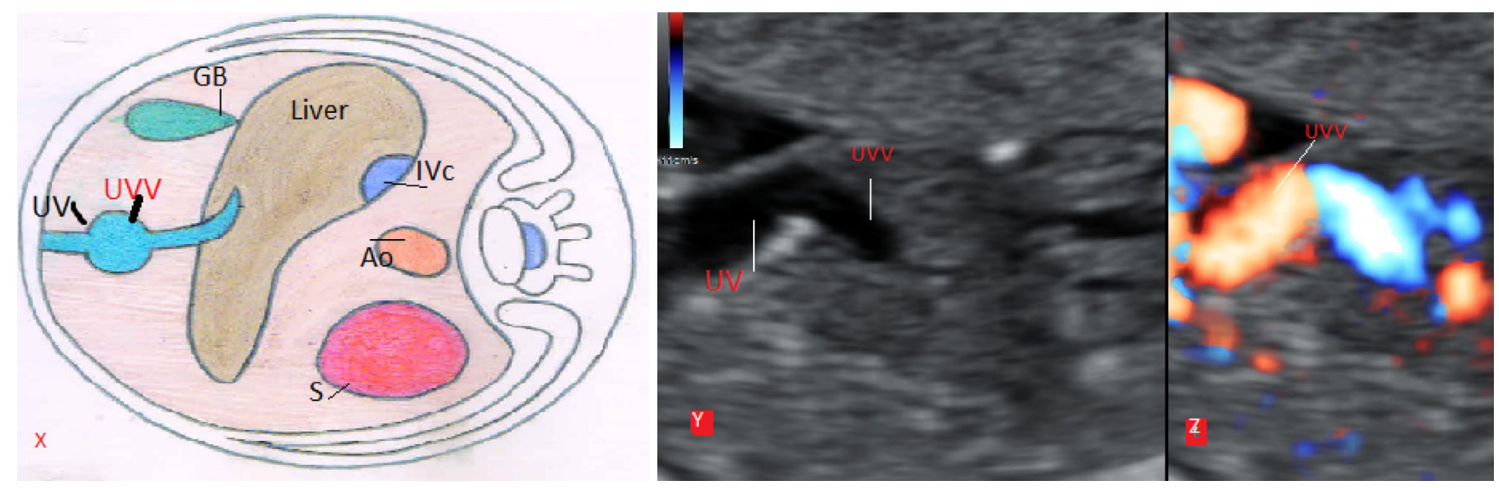

Fig. 2 Schematic drawing (x), two-dimensional (y) and colour doppler (z) images of UVV. Ao, aorta; GB, gall bladder; IVC, inferior vena cava; $\mathrm{S}$, stomach; UV, umbilical vein; UVV, umbilical vein varix

(case 8-12). In all five cases, DV was absent. UPSVS can be broadly categorized in two groups by UV drainage sites:

1. UV not connected to portal vein and drains directly into systemic circulation, called extrahepatic umbilical systemic shunt. We detected three cases (case 8-10) (Fig. 4a-d).

2. UV connects to portal vein, and portal vein then connects to systemic vein, called intrahepatic portal systemic shunt. We detected two cases (case 11, 12) (Fig. 4e, f).

All three cases of extrahepatic umbilical systemic shunt were associated with poor outcome due to cardiac failure and hydrops. In two cases, UV was directly connected to IVC (case 8-9) (Fig. 4a, b) distal to subdiaphragmatic vestibulum and in one case directly to right atrium (case 10) (Fig. 4c, d). In all three cases, intrahepatic portal system was not developed or poorly developed. Two cases of intrahepatic portal systemic shunt had favourable outcome. In both cases, UV was connected to LPV but ductus venosus was absent. Intrahepatic portal system was normally developed. We found connection between right posterior PV to IVC in one case (case 11) (Fig. 4e, f).

\section{Discussion}

Highly oxygenated blood is delivered to left heart by UV, intrahepatic portal venous system and DV. As mentioned in Yagel et al. [2, 3], umbilical vein-portal vein-ductus venosus complex is an integral vascular unit. LPV is the watershed of fetal circulation, meeting point of UV and portal system. Anomalies of UV can be grouped in three main entities: Umbilical vein varix, Persistent right umbilical vein and umbilical portal systemic shunt abnormality (Ductus venosus agenesis). According to Yagel et al. [2,3], the exact prevalence in population is difficult to estimate, some isolated anomalies like PRUV may be asymptomatic while agenesis of ductus venosus and portal venosus system anomalies may have serious sequelae.

\section{Umbilical Vein Varix}

UVV is focal dilatation of UV, it can be intraabdominal or rarely intraamniotic. The diameter usually varies from 8 to $15 \mathrm{~mm}$. It can be defined as intraabdominal UV diameter more than $9 \mathrm{~mm}$ or size 1.5 times larger than intrahepatic UV. Sonographically intraabdominal UVV is seen as anechoic cystic area between cord insertion and liver (Fig. 2). Colour doppler is key to distinguish it from other abdominal cyst. It reveals turbulent flow and continuous with UV. Gestational age at diagnosis, diameter at diagnosis, increase in size of diameter and associated anomalies are prognostic factors. Diagnosis of UVV warrants further detail examination to search for other anomalies, close follow up for fetal growth and thrombus formation. Large varix may lead fetal anaemia and hydrops. According to Wood et al. [4], isolated UVV should be considered benign.

\section{Persistent Right Umbilical Vein}

Usually RUV degenerates during 4-6 embryonic weeks and left persist. Failure of RUV degeneration results into PRUV. Usually only one UV persist. But in some case, both persist and can be found as an intrahepatic supernumerary vein, connecting to the right portal vein. It may also bypass the liver, causing aberrant drainage of blood into systemic circulation. This leads to four vessel cord.

As Weichert et al. [5] have described, PRUV may be isolated anomaly or as a part of complex malformation of viscera and venous system like situs inversus totalis or heterotaxy syndrome.

Normally, during intraabdominal course LUV after joining to LPV turns to right, so the curve is opposite to stomach, gall bladder is located right to UV (Fig. 3a, x). 

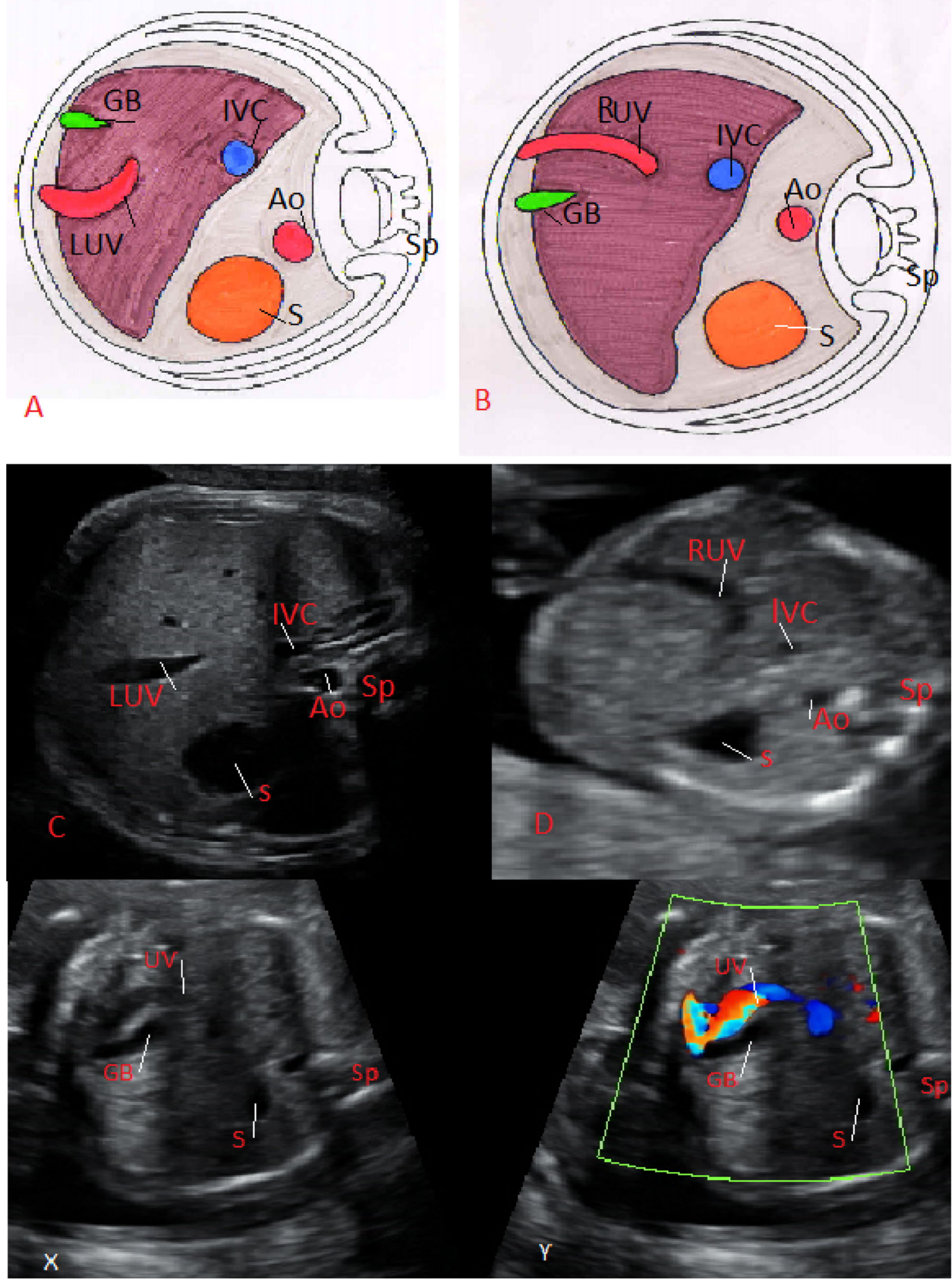

Fig. 3 Schematic drawings of LUV (a) and RUV (b); two-dimensional image of LUV (c) and RUV (d). Axial-oblique two-dimensional (x) and colour doppler (y) images of RUV. Ao, aorta; GB, gall bladder; LUV, left umbilical vein; RUV, right umbilical vein; S, stomach; Sp, spine 
Fig. 4 Schematic drawings (a, c, e), two-dimensional images $(\mathbf{b}, \mathbf{f})$ and colour doppler image (d) of abnormal umbilical portal venosus systemic shunt. Note the absence of ductus venosus (DV). Sh (shunt) from posterior right portal vein (PRPV) to IVC in images F. Ao, aorta; H, heart; $\mathrm{HV}$, hepatic vein; L, liver; LPV, left portal vein; MPV, main portal vein; RAPV, right anterior portal vein; RPV, right portal vein; S, stomach; UV, umbilical vein
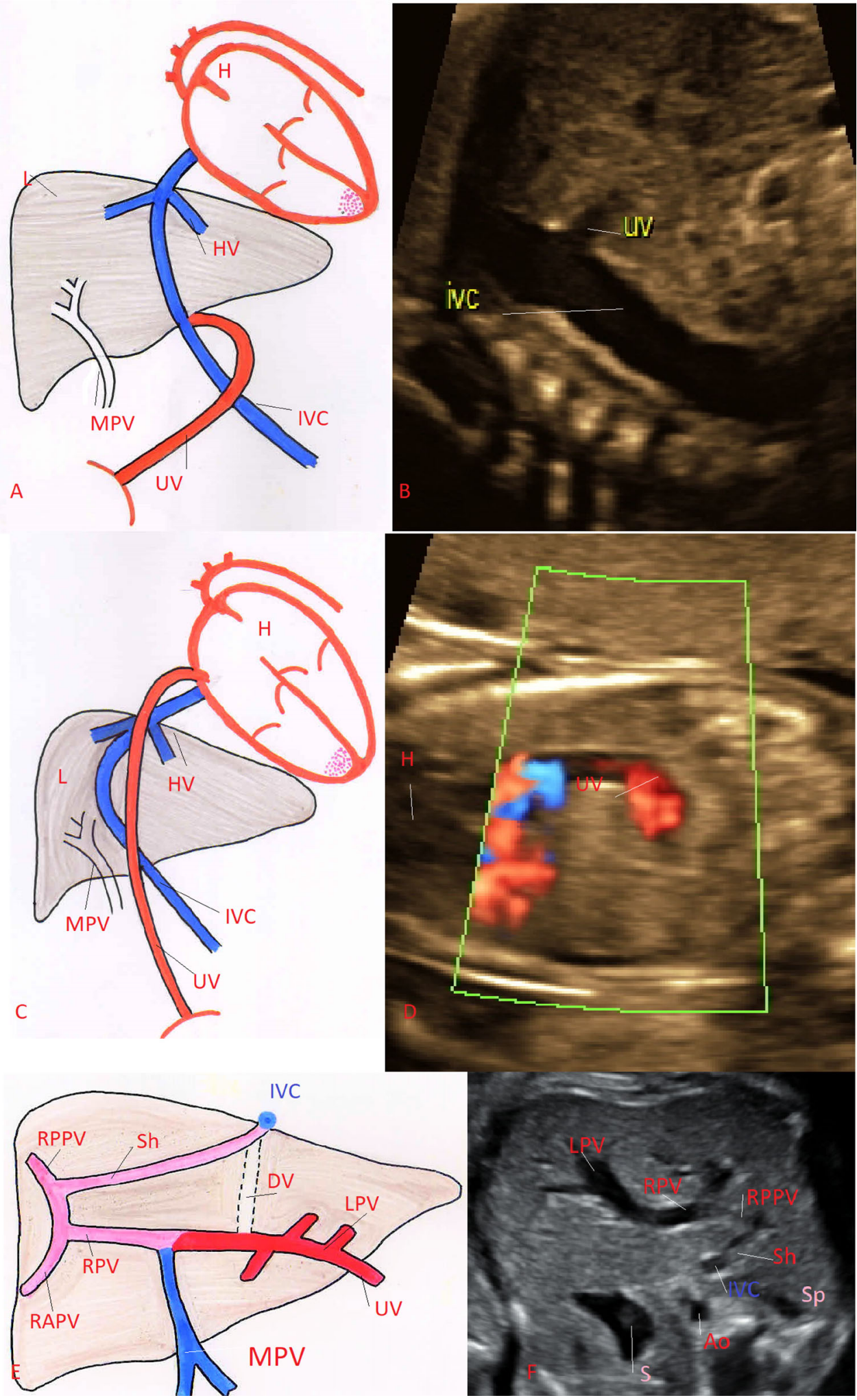

With PRUV, UV connects to right portal vein, curve is towards the stomach and gall bladder is located in between UV and stomach (Fig. 3b, y).
One of our cases was associated with single umbilical artery.

With situs inversus totalis, usually right umbilical vein is normal association. We examined two cases of situs 
inversus totalis, both with normal heart. One with RUV and another with LUV. LUV in this case is considered as persistent left umbilical vein, an anatomical variant, so we have included this case in our study.

Finding of PRUV is an indication for detailed fetal examination and echocardiography to rule out malformations, its portal vein connection and growth monitoring. Prognosis is favourable in isolated cases.

\section{Umbilical Portal Systemic Venous Shunt}

Here, DV agenesis is the key feature. DV is a shunt that preferentially delivers highly oxygenated blood to the left heart. UV connects to LPV, further after giving three branches to left lobe of liver, it continues as portal sinus and gives rise to DV. At 20 weeks around $30 \%$ of blood is shunted through DV and at 30 weeks around 20\%. Small for gestational age fetuses and with increasing placental resistance, DV proportion significantly increases.

DV is a shunt between UV and systemic circulation, it bypasses the liver. With agenesis of DV, abnormal UPSVS develops, here the most important prognostic factor is connection of UV with systemic circulation. Achiron et al. [6] and Stabulidau et al. [7] studied outcome in fetuses with DV agenesis. When UV directly connects to systemic circulation and bypasses the portal system, this leads to cardiac overload and congestive cardiac failure. Simultaneously, intrahepatic portal system fails to develop or partially develop. When UV has connected with the intrahepatic portal venous system, the portal systemic shunt diverts high oxygenated blood to IVC and right atrium. So, this group is further divided into two categories,

1. Extrahepatic umbilical systemic shunt-UV drains directly into right atrium (Fig. 4c, d) or IVC (Fig. 4a, b) or iliac vein or renal vein or coronary sinus etc. Intrahepatic portal system fails to develop or partially develop. Prognosis is poor. Prognosis depends on size of connection between UV and systemic circulation. Few cases where the shunt is narrow like DV, the prognosis may be favourable as it prevents cardiac overload and some portal system may develop.

2. Intrahepatic portal systemic shunt- UV normally connects to PV, intrahepatic PV is connected to IVC by hepatic vein or some vessel as we documented a vessel connecting posterior RPV to IVC (Fig. 4e, f). As Yagel et al. [3] and Achiron et al. [6] described, this group with isolated finding has good outcome.
So, location of UV drainage site is a prognostic factor, cases without liver bypass have better prognosis.

Our study has several limitations. Total number of cases are small, particularly anomalous UPSVS cases. Also, we had not studied extrahepatic portal venous system.

\section{Conclusion}

Examination of UV and DV should be part of routine second trimester anomaly scan. Some rare anomalies like UVV, PRUV and agenesis of DV can be diagnosed. Isolated cases of UVV and PRUV have favourable prognosis. Cases of extrahepatic UPSVS may have poor outcome. Cases of intrahepatic UPSVS have good prognosis.

\section{Compliance with Ethical Standards}

Conflict of interest The authors declare that they have no conflict of interest.

Open Access This article is distributed under the terms of the Creative Commons Attribution 4.0 International License (http://crea tivecommons.org/licenses/by/4.0/), which permits unrestricted use, distribution, and reproduction in any medium, provided you give appropriate credit to the original author(s) and the source, provide a link to the Creative Commons license, and indicate if changes were made.

\section{References}

1. Yagel S, Kivilevitch Z, Cohen SM, et al. The fetal venous system, Part I: normal embryology, anatomy, hemodynamics, ultrasound evaluation and Doppler investigation. Ultrasoud Obstet Gynecol. 2010;35(6):741-50.

2. Yagel S, Cohen SM, Valsky DV, et al. Systematic examination of the fetal abdominal precordial veins: a cohort study. Ultrasoud Obstet Gynecol. 2015;45:578-83.

3. Yagel S, Kivilevitch Z, Cohen SM, et al. The fetal venous system, Part II: ultrasound evaluation of the fetus with congenital venous system malformation or developing circulatory compromise. Ultrasound Obstet Gynecol. 2010;36(1):93-111.

4. Wood DC, Giannone M, Gammal E, et al. Isolated intra-abdominal umbilical vein (IAUV) varix in 163 fetuses: antenatal management and postnatal outcomes. Ultrasoud Obstet Gynecol. 2010;36:156.

5. Weichert J, Hartge D, Germer U, et al. Persistent right umbilical vein: a prenatal condition worth mentioning? Ultrasound Obstet Gynecol. 2011;37:543-8.

6. Achiron R, Kivilevitch Z. Fetal umbilical-portal-systemic venous shunt: in-utero classification and clinical significance. Ultrasound Obstet Gynecol. 2016;47(6):739-47.

7. Stabulidau I, Pereira S, Cruz Jde J, et al. Prevalence and outcome of absence ductus venosus at $11(+0)$ to $13(+6)$ weeks. Fetal Diagn Ther. 2011;30(1):35-40. 